\title{
Effectiveness of Differential Reinforcement of Other Behaviors in Reducing Non- suicidal Self-Injurious Behaviors in Adolescents
}

\author{
Fatimah Nosrati ${ }^{1}{ }^{*}$, Ali Massah Bavani ${ }^{2}$ and Bagher Ghobari Bonab ${ }^{3}$ \\ ${ }^{1}$ Assistant Professor, School of Psychology and Education, University of Tehran, Tehran, Iran \\ 2MA, School of Psychology and Education, University of Tehran, Tehran, Iran \\ ${ }^{3}$ Professor, School of Psychology and Education, University of Tehran, Tehran, Iran
}

* Corresponding author: Fatimah Nosrati, School of Psychology and Education, University of Tehran, Tehran, Iran. Tel: +989121250558;

Email: fnosrati@ut.ac.ir

Received 2021 March 02; Revised 2021 May 16; Accepted 2021 September 19.

\begin{abstract}
Background: Non-suicidal self-injury is a prevalent phenomenon, especially among adolescents, with substantial personal and social consequences. To reduce such behaviors, differential reinforcement of other behaviors has gained substantial empirical support. However, previous investigations on the efficacy of this approach have mostly focused on individuals with developmental disorders, and it is still unknown whether such an approach is a viable treatment option for typically developed individuals.

Objectives: This study aimed to investigate the effectiveness of differential reinforcement of other behaviors in reducing non-suicidal self-injury behaviors in typically developed adolescents.

Methods: A single-subject A-B-A-B design was used in this study. The statistical population included male adolescents with self-injurious behaviors, the families of whom were seeking treatment for these behaviors. In total, four adolescents were recruited from a psychology clinic in Tehran, Iran, using convenience sampling. Participants were observed at 6, 8, 10, and 12 sessions at baseline phases of $\mathrm{A}_{1}$ and $\mathrm{A}_{2}$, followed by 12 intervention sessions after each baseline phase. The intervention included differential reinforcement of other behaviors. If the participants showed no self-injury behavior within a specific time duration, a reward was provided. Non-suicidal self-injury behaviors included self-harm, hair pulling, severe itching, pinching, wound manipulation, and hand biting. The frequency of these behaviors was assessed during each session. Visual analysis of graphed data, percentage of non-overlapping data, and mean percentage improvement were used for data analysis.

Results: The results showed a fairly reliable effect for the intervention on reducing the target behavior, as indicated by a frequency reduction from phase $A_{1}$ to $B_{1}$ and $A_{2}$ to $B_{2}$ and by a frequency elevation by the intervention withdrawal from $B_{1}$ to $A_{2}$. The average percentage reduction across participants was obtained at $56 \%$. However, a low rate of self-injury remained consistent for the participants. Conclusion: The results provided further evidence on the effectiveness of differential reinforcement of other behaviors in reducing selfinjury behaviors. Although the intervention could reduce self-injury substantially, it seemed that it could not eliminate the behavior.

Keywords: Behavior therapy, Problem behavior, Reinforcement, Self-injurious behavior, Token economy
\end{abstract}

\section{Background}

Non-suicidal self-injury disorder is presented in Section 3 of the Diagnostic and Statistical Manual of Mental Disorders (1) to be a subject for further investigations. The condition features engagement in bodily self-injury without suicide intention that leads to a mild or moderate physical injury occurring at least 5 days a year and causing distress in a person's daily life. Based on the proposed diagnostic criteria, the person engages in such behaviors to relieve negative feelings, solve some interpersonal problems, or induce positive feelings.

Non-suicidal self-injury disorder is more prevalent in adolescents (2). In an international investigation on a representative sample from 11 European countries, the lifetime prevalence rate was obtained at $28 \%$ (3). Moreover, the lifetime prevalence of self-injury behaviors in the US normal population was estimated at $5.9 \%$ (4). Based on international data, the average lifetime prevalence in adolescents is $18 \%$ (5). In another study conducted in Belgium, Buelens et al. (6) examined the proposed diagnostic criteria for non-suicidal self-injury behaviors in a sample of adolescents. The results showed that the prevalence rate of non-suicidal selfinjury was $17.6 \%$, and this rate was higher in girls than boys. In Iran, while nationwide estimates are seemingly lacking, some individual studies have estimated the lifetime prevalence rate at $12 \%$ among university students (7) and about 6\% among highschool students (8), which is substantially lower than average international estimates $(3,5)$. However, adolescence-onset is considerably high. A recent large-scale longitudinal study $(n=6229)$ showed an onset rate of $3.6 \%$ among high-school students within a one-year period (8).

Since non-suicidal self-injury is most prevalent among adolescents, the majority of the investigations have focused on this age group. In adolescents, nonsuicidal self-injury behaviors are not only limited to direct self-harm behaviors, such as amputation, biting, scratching, and burning but they also include indirect self-harm behaviors, including recklessness, harmful eating behaviors, and drug abuse (9).

It is also worth noting that non-suicidal self-injury 
is often associated with both internalizing and externalizing disorders (10). While self-injury behaviors serve some short-term functions for the person (11), it is often associated with long-term negative physical, emotional, and social consequences $(12,13)$. In the international study that was mentioned earlier, suicidality, depression, anxiety, and loneliness/interpersonal problems had the strongest associations with non-suicidal selfinjury (3). Notably, in this study, only a minority of the adolescents with this condition ever received medical treatment. Along with the risks associated with these behaviors and the psychological and social consequences for the individuals who engage in such behaviors, non-suicidal self-injury also imposes substantial stress on parents (14). These characteristics and consequences highlight the need for interventions that can address the problem.

To reduce self-injurious behaviors, different psychological approaches have been proposed and investigated, including cognitive-behavioral therapy (CBT), dialectical behavioral therapy (DBT), parent training, and interpersonal psychotherapy. Current empirical data seem to support the overall effect of these interventions; however, it does not provide sufficient evidence on the superiority of a specific approach $(15,16)$. On the other hand, in addition to these approaches that mostly focus on cognition, the utilization of behavioral principles has been proposed, and there is substantial evidence on their effectiveness in reducing self-injurious behaviors, especially in children with developmental disorders.

In an influential study, Kahng et al. meta-analyzed 396 studies conducted between 1964 and 2000 on the effectiveness of different behavioral interventions in reducing self-injury among individuals with developmental disorders. They reported an average reduction of $84 \%$ in self-injurious behaviors across interventions (17). An update was recently published which included 94 additional studies conducted between 2001 and 2016 and reported a shift from intellectual disability toward autism spectrum disorders (18). Moreover, in this update, the estimated efficacy for the new studies was lower (67\% reduction on average) maybe because the majority of the studies were conducted on participants with automatically reinforced selfinjurious behavior (as opposed to socially reinforced self-injurious behaviors), which is a specific subtype that constitutes roughly one-fourth of cases with possibly different treatment responses (19). Furthermore, Morano et al. (20) conducted a metaanalysis on 137 single-subject studies to evaluate the effectiveness of various intervention approaches in reducing self-injurious behaviors among individuals with autism and/or intellectual disabilities. While the overall results suggested that the various interventions are effective, the largest effect size was for reinforcement- and/or punishment-based interventions. Following these results and due to the invasive nature of punishment, the author recommended reinforcement-based interventions for all cases with self-injurious behaviors.

Differential reinforcement is of behavioral technique, which has been utilized to reduce selfinjurious behaviors and has gained considerable empirical support, mainly from single-subject investigations $(17,18,20)$. Differential reinforcement for self-injury has been used and investigated usually in the form of either differential reinforcement of alternative behavior (DRA) or differential reinforcement of other behavior (DRO).

DRA and DRO directly utilize the most basic processes of learning (i.e., the behavioral principles), and they are expected to be beneficial within a wide range of conditions and populations. Furthermore, because of the simplicity of the procedure (albeit after designing the treatment plan), these interventions can be delivered by parents, making the intervention less costly and more flexible for practical utility. However, while previous studies have provided substantial evidence on the effectiveness of various behavioral interventions, including DRA and DRO, they mostly focused on individuals with developmental disabilities, especially intellectual disability and autism. However, non-suicidal self-injury is not exclusive to patients with mental disorders.

\section{Objectives}

In the proposed diagnostic criteria for the nonsuicidal self-injury disorder, it is stated that the condition should not be "better explained by another mental disorder or medical condition" (1). This study aimed to investigate the effectiveness of DRO in reducing self-injurious behaviors in typically developed male adolescents.

\section{Methods}

\subsection{Design}

The study was conducted under the support of the Art of Life, a psychology clinic in Tehran, Iran. An AB-A-B single-subject design was used in this study $(21,22)$. At the first stage $\left(A_{1}\right)$, the baseline frequency of behavior was measured on several occasions $(6,8$, 10 , and 12 occasions for the first through the fourth participant, respectively). At stage $B_{1}$, the experimental operation was performed, and the frequency of the target behaviors was measured (12 occasions). The second baseline stage $\left(\mathrm{A}_{2}\right)$ was started about one month after the end of phase $B_{1}$, and the frequency of self-injury was measured $(6,8$, 10 , and 12 measurement occasions similar to the $A_{1}$ phase). At the final stage $\left(\mathrm{B}_{2}\right)$, the experimental operation was repeated and measured (12 occasions). 


\subsection{Participants}

Male adolescents with an age range of 13 to 17 years and 11 months along with self-injury behaviors were eligible to be included in the study. Self-injury behavior was defined as each of the 12 types of selfinjury behaviors described by Klonsky and Glenn (23) that have been done consciously and intentionally but not with suicidal intention (see the Measure subheading below). No additional criteria were considered. In total, four participants were recruited through the convenience sampling method.

All participants were physically healthy. Participant 1 was a 13-year-old boy, who had a younger sister. His primary self-injury behavior was in the form of biting his hands. There were sores on both his wrists and between his index and middle fingers. In addition to biting his hand, he was also hit his head with his hand; however, because of its very low frequency, it was not considered a target behavior.

Participant 2 was 16 years old. He was born from his mother's second marriage, and when he was 2 years old, his parents separated. He spent his childhood some time with his mother and some time with his stepmother. However, during this study, he was living with his mother, and he had 2 half-sisters and 3 half-brothers, one of whom had schizophrenia. His self-injury was hitting his head and face with his hands along with visible wounds on his body.

Participant 3 was 13 years old and grew up in an educated family. He was reluctant to study and admitted that his parents' pressures had made him tired of studying. Hussein was physically healthy, and his self-injury behaviors were in the form of pinching his body so that bruises could be seen in different parts of his body.

Participant 4 was 14 years old. He was the last child. At age 12, he had lost his uncle and after this incident, he had suffered from a depressed mood for a long time. His self-injury behavior was in the form of hitting himself, which once led to a scalp fracture.

\subsection{Measure}

To assess the behavior and functions of nonsuicidal self-injury, the Inventory of Statements About Self-injury (ISAS; Klonsky and Glenn, 2009) was used, which assesses 12 types of self-injury behaviors, such as biting, banging/hitting self, needle-sticking, pinching, cutting, wound picking, burning, carving, severe scratching, rubbing skin against rough surfaces, hair pulling, and swallowing chemicals. ISAS also assesses the functions of these behaviors. The inventory has been translated to Persian and has shown acceptable validity (convergent and divergent), test-retest reliability (intra-class correlation=0.76), and internal consistency $(\alpha=0.93)(24)$.

\subsection{Intervention}

Initially, strong and effective reinforces were identified for each participant by asking the participants' instructors or parents. For participant 1 , the favorite cookie was considered reinforcement; however, to prevent food satiation that reduces the reward value, the cookies were presented in small pieces. For participant 2, 10 Rials coins were selected due to the individual's interest in coin collecting. For participants 3 and 4, coupons were presented, each of which would allow the person to play for 5 minutes with a Smartphone or PlayStation, respectively.

The baseline phase $\left(A_{1}\right)$ data were utilized to specify the appropriate time duration to define successful reinforcement achievement. Since the intervention was to reinforce every behavior other than self-injury, it was necessary to determine which time duration could be considered the achievement of engaging in other behaviors, while restraining from self-injury. At the baseline, the time intervals between self-injury behaviors were found to be mostly between 5 and 8 minutes depending on the participants. Every time the participants did not show self-injury behavior for the specified time duration, reinforcement would be provided. This procedure is assumed to reduce the problem behavior since the participant tries to receive the reinforcement by the abstinence of the problem behavior.

\subsection{Analysis}

Visual analysis of the plotted data was considered the primary method for data analysis. Furthermore, the Percentage of Non-overlapping Data (PND) was calculated for each participant as effect size (25).

\section{Results}

Table 1 summarizes the statistics for self-injury behaviors, and individual data points are plotted in Figure 1 . The mean number of self-injury behaviors across phase $A_{1}$ varied among the participants from 7.5 to 17 with a mean percentage reduction of $56 \%$. Participant 3 showed substantially more frequent self-injury behaviors than the other three participants with a minimum number of 13 . As shown in Figure 1, at the first three sessions, participant 3 showed a very high frequency in self-injury behaviors which declined through this phase and then became fairly fixed. This pattern is, probably, due to unfamiliarity with the setting at the starting sessions. However, the other three participants did not show any increasing or decreasing trend, rather they showed random variation between sessions.

At $B_{1}$, the average frequency for all participants decreased to approximately one-half or less. The trend line showed a steep decreasing slope for all participants with some random variation. The effect seems reliable for participants 3 and 4 since the random variation is low, and there is no overlapping data point (both PNDs=100). For participants 1 and 2, though it is not as clear as that for the other two 
Table 1. Summary of Statistics and Intervention Effect-Sizes on the Frequency of Self-Injury Behaviors

\begin{tabular}{|c|c|c|c|c|}
\hline & Participant 1 & Participant 2 & Participant 3 & Participant 4 \\
\hline \multicolumn{5}{|l|}{ Phase $\mathbf{A}_{1}$} \\
\hline Mean & 10.5 & 7.5 & 17 & 9.42 \\
\hline Median & 11 & 7.5 & 17 & 9 \\
\hline Minimum & 8 & 4 & 13 & 8 \\
\hline Maximum & 12 & 12 & 22 & 12 \\
\hline \multicolumn{5}{|l|}{ Phase $B_{1}$} \\
\hline Mean & 5.75 & 3.25 & 8.08 & 3.67 \\
\hline Median & 6.5 & 3 & 8 & 4 \\
\hline Minimum & 3 & 1 & 6 & 1 \\
\hline Maximum & 8 & 8 & 10 & 7 \\
\hline \multicolumn{5}{|l|}{ Phase $\mathrm{A}_{2}$} \\
\hline Mean & 9.17 & 5 & 14.3 & 6.42 \\
\hline Median & 9 & 5 & 14 & 7 \\
\hline Minimum & 8 & 2 & 11 & 4 \\
\hline Maximum & 7 & 17 & 9 & 17 \\
\hline \multicolumn{5}{|l|}{ Phase $B_{2}$} \\
\hline Mean & 3.08 & 1.42 & 5.08 & 1.92 \\
\hline Median & 4 & 1 & 5 & 2 \\
\hline Minimum & 0 & 0 & 4 & 1 \\
\hline Maximum & 3 & 8 & 3 & 1 \\
\hline \multicolumn{5}{|c|}{ PND Across Phases } \\
\hline$B_{1}$ vs. $A_{1}$ & 83 & 67 & 100 & 100 \\
\hline $\mathbf{B}_{2}$ vs. $A_{2}$ & 100 & 58 & 100 & 100 \\
\hline \multicolumn{5}{|c|}{ Mean Percentage Improvement Across Phases(1) } \\
\hline$B_{1}$ vs. $A_{1}$ & 45 & 40 & 85 & 55 \\
\hline$B_{2}$ vs. $A_{2}$ & 58 & 34 & 88 & 43 \\
\hline
\end{tabular}

Note. The numbers of measurements at phases $A_{1}$ and $A_{2}$ were $6,8,10$, and 12 for Participants 1 through 4 , respectively. The number of measurements at phases $\mathrm{B}_{1}$ and $\mathrm{B}_{2}$ was 12 for all participants.

PND=Percentage of non-overlapping data.

(1) Averaging the mean percentage improvement from both phase changes across participates showed an overall 56\% reduction in selfinjurious behaviors.

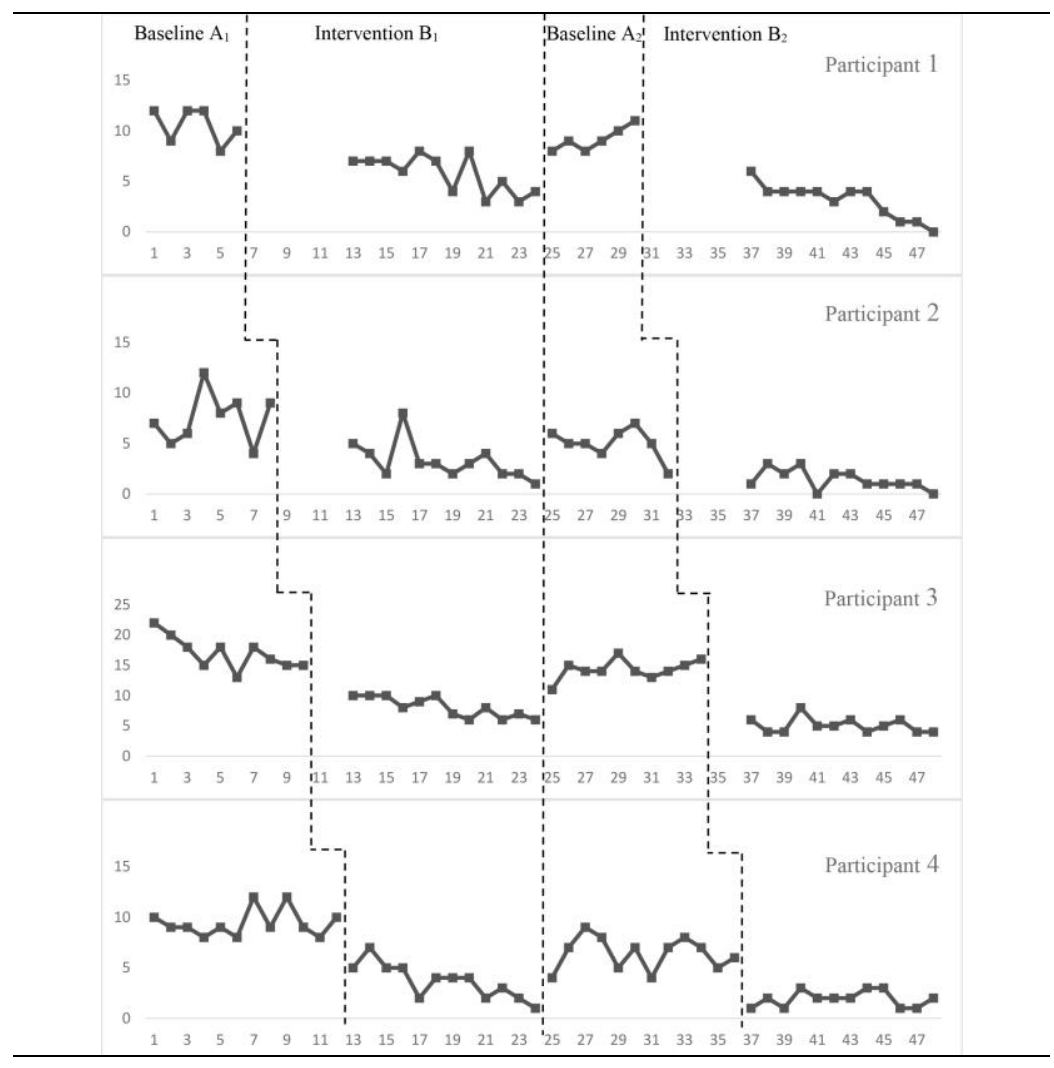

Figure 1. Frequency of participants self-injury behaviors across the four phases

participants, the effect seems fairly reliable (Figure 1 and the PNDs in Table 1). However, the PND for participant 2 was 67 which is usually categorized as indicating a questionable effect.

At phase $A_{2}$ in which the intervention was withdrawn, the average frequency increased again 
considerably; however, it was not as much as that in phase $A_{1}$. This provides further evidence on the effect of the intervention at phase $B_{1}$ as the frequency of self-injury increased after intervention withdrawal.

Finally, at phase $B_{2}$, the frequency of self-injury behaviors declined again and reached the lowest level across all phases. The mean percentage reduction was similar to the first intervention phase (56\%). Except for participant 2, the effect seemed reliable, as indicated by the trend lines in Figure 1, and that there was no overlapping data point between $A_{2}$ and $B_{2} \quad(P N D=100)$. However, for participant 2, the effect is not as clear, especially as the frequency in $B_{2}$ which is similar to the frequency in the last $A_{2}$ data point.

\section{Discussion}

This study used a single subject A-B-A-B design to evaluate the effectiveness of DRO in reducing selfinjury behaviors in male adolescents. Participants were observed for several one-hour sessions, in which the frequency of their self-injurious behaviors was recorded. This was followed by a 12 -session intervention phase. After a one-month interval, the same A-B sequence was repeated. The results showed a fairly reliable effect for the intervention on reducing the target behavior, as indicated by a frequency reduction from phase $A_{1}$ to $B_{1}$ and $A_{2}$ to $B_{2}$ and by a frequency elevation by the intervention withdrawal from $B_{1}$ to $A_{2}$. For three of the four participants, the average baseline frequency was between 7.5 and 10.5. At the ending sessions of the second intervention phase, the frequency approached zero (between 0 and 2 in the last three sessions of $B_{2}$ ). For the other participants who showed a very high rate of self-injury at baseline (on average 17 at $A_{1}$ ), the frequency declined to one-fourth in the last two intervention sessions. Importantly, while the intervention reduced the target behavior, it did not eliminate the behavior, rather it became relatively stable at a low rate at least for two participants.

The results from this study are in line with the previous findings from studies conducted on individuals with autism and intellectual disability in that DRO was highly effective in reducing self-injury behaviors $(17,18,20)$. The current study suggests that while DRO has been mostly used in individuals with developmental disorders, its effectiveness may not be limited to this population. However, unlike individuals with developmental disorders, especially individuals with intellectual disabilities, there are various evidence-based treatment options available for typically developed individuals $(15,16)$. In comparison to the other common psychosocial interventions, such as CBT and DBT, differential reinforcement provides an effective treatment method with less cognitive demand. It affects behavior as it utilizes the most basic processes that are at the core of human learning without demanding more complicated higher cognitive abilities, such as complicated reasoning and problem-solving. However, it is important to note the basic difference between these approaches. While pure behavioral techniques aim only for the problem behavior itself, most other psychosocial approaches consider the problem as the manifestation of some underlying factors that usually assume to be involved in more than just self-injurious behaviors. This difference can lead to narrowed effects for pure behavioral treatments and more general effects for other psychosocial approaches. However, it is yet not clear that which approach, under what conditions, and for whom is more effective. To answer these questions, further comparative investigations are warranted, as well as investigations on treatment outcome moderators.

Another important finding was the repeatedly observed effectiveness limitation that the interventions do not usually eliminate self-injurious behaviors entirely $(17,18,20)$. In this study, during the intervention sessions in which they were receiving immediate rewards for abstinence, they were still showing self-injurious behaviors, though in low-rate frequency. It is worth noting that two participants had a zero frequency of self-injury in their last intervention sessions; however, it was unclear whether these were stable changes or just random variations. The maintenance of low-rate selfinjury may be considered a challenge for theoretical accounts of non-suicidal self-injury. It also may pose some practical implications, and when self-injurious behaviors have not been eliminated, the functional analysis may be necessary for preventing the remained problem behaviors that receive further reinforcement.

\section{Conclusion}

The results provided further evidence for the effectiveness of the reinforcement of other behaviors in reducing self-injurious behaviors in male adolescents with no developmental disorder. Although the investigations on the effectiveness of the behavioral techniques on non-suicidal self-injury in individuals without developmental disorders are rare, this study suggests promising results. Further research is warranted to investigate the possible utility of these methods in environments, such as correctional centers, where the rate of non-suicidal self-injury behavior is high.

\section{Acknowledgments}

None.

\section{Footnotes}

Authors' contributions: This study was extracted 
from an MSc thesis written by A. M. B. with the supervision of F. N. and the methodological consultant of B. G. B. The research was conducted by A. M. B and the manuscript was written by F. N.

Ethical Approval: The study protocol was approved by the Educational Council of the Faculty of Psychology and Educational Sciences, University of Tehran, Tehran, Iran (IR.UT.PSYEDU.REC.1399.035).

\section{Funding/Support: None}

Financial Disclosure: None

Conflicts of Interest: The authors had no conflict of interest to declare.

\section{References}

1. Battle DE. Diagnostic and statistical manual of mental disorders (DSM). Codas. 2013;25(2):191-2. doi: 10.1590/s2317-17822013000200017. [PubMed: 24413388].

2. Zetterqvist M. The DSM-5 diagnosis of nonsuicidal self-injury disorder: a review of the empirical literature. Child Adolesc Psychiatry Ment Health. 2015;9(1):31. doi: 10.1186/s13034015-0062-7. [PubMed: 26417387].

3. Brunner R, Kaess M, Parzer P, Fischer G, Carli V, Hoven CW, et al. Life-time prevalence and psychosocial correlates of adolescent direct self-injurious behavior: a comparative study of findings in 11 European countries. J Child Psychol Psychiatry. 2014;55(4):337-48. doi: 10.1111/jcpp.12166. [PubMed: 24215434].

4. Klonsky ED. Non-suicidal self-injury in United States adults: prevalence, sociodemographics, topography and functions. Psychol Med. 2011;41(9):1981-6. doi: 10.1017/S0033291710002497. [PubMed: 21208494].

5. Muehlenkamp JJ, Claes L, Havertape L, Plener PL. International prevalence of adolescent non-suicidal self-injury and deliberate self-harm. Child Adolesc Psychiatry Ment Health. 2012;6(1):10. doi: 10.1186/1753-2000-6-10. [PubMed: 22462815].

6. Buelens T, Luyckx K, Kiekens G, Gandhi A, Muehlenkamp JJ, Claes L. Investigating the DSM-5 criteria for non-suicidal selfinjury disorder in a community sample of adolescents. J Affect Disord. 2020;260:314-22. doi: 10.1016/j.jad.2019.09.009. [PubMed: 31521868].

7. Gholamrezaei $M$, Heath N, Panaghi L. Non-suicidal self-injury in a sample of university students in Tehran, Iran: prevalence, characteristics and risk factors. Int J Cult Ment Health. 2017;10(2):136-49. doi: 10.1080/17542863.2016.1265999.

8. Marin S, Hajizadeh M, Sahebihagh MH, Nemati H, Ataeiasl M, Anbarlouei $\mathrm{M}$, et al. Epidemiology and determinants of selfinjury among high school students in Iran: a longitudinal study. Psychiatr Q. 2020;91(4):1407-13. doi: 10.1007/s11126020-09764-z. [PubMed: 32418140].

9. Cassels $M$, Wilkinson $P$. Non-suicidal self-injury in adolescence. Paediatr Child Health. 2016;26(12):554-8. doi: 10.1016/j.paed.2016.08.006.

10. Meszaros G, Horvath LO, Balazs J. Self-injury and externalizing pathology: a systematic literature review. BMC Psychiatry. 2017;17(1):160. doi: 10.1186/s12888-017-1326-y. [PubMed: 28468644].
11. Hepp J, Carpenter RW, Störkel LM, Schmitz SE, Schmahl C, Niedtfeld I. A systematic review of daily life studies on nonsuicidal self-injury based on the four-function model. Clin Psychol Rev. 2020;82:101888. doi: 10.1016/j.cpr.2020.101888. [PubMed: 32949907].

12. Wilkinson P, Goodyer I. Non-suicidal self-injury. Eur Child Adolesc Psychiatry. 2011;20(2):103-8. doi: 10.1007/s00787010-0156-y. [PubMed: 21222215].

13. Staniland L, Hasking P, Boyes M, Lewis S. Stigma and nonsuicidal self-injury: Application of a conceptual framework. Stigma Heal. 2021;6(3):312-23. doi: 10.1037/sah0000257.

14. Whitlock J, Lloyd-Richardson E, Fisseha F, Bates T. Parental secondary stress: the often hidden consequences of nonsuicidal self-injury in youth. J Clin Psychol. 2018;74(1):17896. doi: 10.1002/jclp.22488. [PubMed: 28493555].

15. Glenn CR, Franklin JC, Nock MK. Evidence-based psychosocial treatments for self-injurious thoughts and behaviors in youth. J Clin Child Adolesc Psychol. 2015;44(1):1-29. doi: 10.1080/15374416.2014.945211. [PubMed: 25256034].

16. Plener PL, Brunner R, Fegert JM, Groschwitz RC, In-Albon T, Kaess M, et al. Treating nonsuicidal self-injury (NSSI) in adolescents: consensus based German guidelines. Child Adolesc Psychiatry Ment Health. 2016;10(1):46. doi: 10.1186/s13034016-0134-3. [PubMed: 27933099].

17. Kahng S, Iwata BA, Lewin AB. Behavioral treatment of selfinjury, 1964 to 2000. Am J Ment Retard. 2002;107(3):212-21. doi: $10.1352 / 0895-8017(2002) 107<0212$ :BTOSIT>2.0.CO;2. [PubMed: 11966334].

18. Shawler LA, Russo SR, Hilton JL, Kahng S, Davis CJ, Dorsey MF. Behavioral treatment of self-injury: 2001 to 2016. Am J Intellect Dev Disabil. 2019;124(5):450-69. doi: 10.1352/19447558-124.5.450. [PubMed: 31512946]

19. Hagopian LP, Rooker GW, Zarcone JR. Delineating subtypes of self-injurious behavior maintained by automatic reinforcement. J Appl Behav Anal. 2015;48(3):523-43. doi: 10.1002/jaba.236. [PubMed: 26223959].

20. Morano S, Ruiz S, Hwang J, Wertalik JL, Moeller J, Karal MA, et al Meta-analysis of single-case treatment effects on self-injurious behavior for individuals with autism and intellectual disabilities. Autism Dev Lang Impair. 2017;2:239694151668839. doi: $10.1177 / 2396941516688399$.

21. Gast DL, Ledford JR, Severini KE. Withdrawal and reversal designs. Single Case Research Methodology. London: Routledge; 2018.

22. Tate RL, Perdices M. Single-case experimental designs for clinical research and neurorehabilitation settings. London: Routledge; 2019. doi: 10.4324/9780429488184.

23. Klonsky ED, Glenn CR. Assessing the functions of non-suicidal self-injury: psychometric properties of the inventory of statements about self-injury (ISAS). J Psychopathol Behav Assess. 2009;31(3):215-9. doi: 10.1007/s10862-008-9107-z. [PubMed: 29269992].

24. Zarghami M, Babakhanian $M$, Habibi Asgarabad $M$, Ghazanfanpour M, Akrami FS, Nazeri N, et al. Psychometric properties of the inventory of statements about self-injury (ISAS) in Iranian opioid and alcohol abusers. Iran J Psychiatry Behav Sci. 2020;14(4):e88494. doi: 10.5812/ijpbs.88494.

25. Scruggs TE, Mastropieri MA. PND at 25: Past, present, and future trends in summarizing single-subject research. Remedial Spec Educ. 2013;34(1):9-19. doi: 10.1177/0741932512440730. 